\title{
Stabilization of A Two-DOF Gimbal System Using Direct Self-Tuning Regulator
}

\author{
Amir Naderolasli ${ }^{1}$ and Mohammad Ataei $^{2}$ \\ ${ }^{1}$ Sama Technical and Vocational Training College, Islamic Azad University, \\ Najafabad Branch, Najafabad, Iran. \\ ${ }^{2}$ Department of Electrical Engineering, Faculty of Engineering, University of Isfahan, \\ Isfahan, Iran. \\ naderolasli@sel.iaun.ac.ir.
}

\begin{abstract}
This paper aims to present the stabilization a two-axis gimbal system. The coupling effects and instances of mass unbalance have been considered in the proposed gimbal model; and a direct self-tuning regulator (STR) is utilized to improve the system performance. For this purpose, the parameters of controller polynomials have been calculated through the application of a recursive least square approach. Alternatively, a specific control signal is generated for both elevation and azimuth axes and applied to the gimbal system. The stabilization results of the targeted two-axis gimbal system confirm the effectiveness of the proposed controller for both elevation and azimuth axes. Moreover, an experimental study is implemented on the dynamics modeling and control of a two-DOF gimbal system. The controller algorithm is implemented through Arduino Uno because of its easy compatibility and portability.
\end{abstract}

Keywords: Stabilization, Two-DOF gimbal system, Self-tuning regulator (STR), Recursive least square (RLS).

\section{Introduction}

Two axis gimbal systems are usually adopted to provide stability for the sensors when different disturbance effects may produce undesirable consequences influencing the operation of the systems. As such, the gimbal system scans for light axis sensors in the azimuth and elevation frames attached to a line which is called the line of sight (LOS). Clearly, the most important sources of disturbance in such systems are the angular situations, the friction between frames, cable restraint, and mass unbalance [1]. The mass unbalance is a serious and inevitable problem in the gimbal systems no matter how accurately they are designed. In point of fact, the mass unbalance causes a torque disturbance in the gimbal system when the body is rotating or accelerating. The frames are originally positioned on one another and form the center of rotation and it is assumed that the gimbal should have no unbalanced mass [2-3]. To control the gimbal system and maintain stability, the operational conditions of the system especially its angular velocity should be monitored. In other words, it is necessary to consider system relevant dynamics to analytically analyze the system before it is actually designed and produced. Accordingly, a common system comprising an electromechanical gimbal with one degree of freedom, two or more light electrical axes are required. The controlling of LOS stabilizer systems is very complicated because of the extant cross coupling between different channels [4]. In addition, these systems are usually required to be kept stabilized in order to guarantee the accuracy of following and track of the targets when there are changes in the dynamics of the system and operational conditions. It is clear that the system performance depends largely on the accuracy of modeling, which is a prerequisite in such systems as lighting equipment including IR, Laser, radar, TV, all widely applied in most important operations like image processing, missile instruction, navigation systems as well as seeker systems [5].

This system can rotate on two elevation and azimuth axes with two gyros, one installed on the other. In fact, these gyros are applied to get feedback from angular velocities to stabilize the inner gimbal frame and prevent it from producing any disturbance in the gimbal body. Notably,

Received: November $17^{\text {th }}, 2018$. Accepted: March $3^{\text {rd }}, 2020$ DOI: 10.15676/ijeei.2020.12.1.3 
it is possible to create a LOS in $3 \mathrm{D}$ spaces through adjusting the input and output frames of the gimbal in relation to the outer frames and gimbal body. The amount of deviation thus produced can be determined for any moving target using image processing systems with a camera installed on the axis of the inner gimbal by [6]. The problem of gimbal system control is the cross coupling between two control loops. The cross coupling can describe the characteristics of dynamic gimbal system and its reflection when azimuth gimbal affects elevation gimbal even when its body is nonrotating [7].

A robust control is suggested for stabilization and control of two-axis gimbal system [8]. A robust PI controller is applied to the system for enhancing high performance and appreciable stabilization [9]. An adaptive fractional-order sliding mode controller is applied for stabilization of two-DOF gimbal system [10]. The inverse system method with the inertial model control is utilized because of the nonlinearity and coupling terms in this system [11]. To enhance the tracking and stabilization of the ISP, an adaptive decoupling control based on neural network is suggested [12]. To guarantee design stabilization, the fuzzy controller is proposed for the twoaxis gimbal system [13]. An adaptive feedback linearization is utilized for stabilization of these systems [14].

On the basis of the above, the present article is organized as follow: Dynamic modeling of two-DOF gimbal system is presented in section 2. In section 3, the direct self-tuning regulator is designed for stabilization of two-DOF gimbal system. The experimental discussions are presented in section 4. Simulation results of two-axis gimbal system are given in section 5 and finally in section 6 , some conclusions are provided.

\section{Dynamic modelling of two-DOF gimbal system}

Two degree of freedom gimbal model with a seeker system is composed of two systems called inner and outer gimbals. In order to control and navigate seeker systems in elevation and azimuth axes, two controlling loops, stabilizer and tracker, have been used. The stabilizer loop stabilizes the axes of tracker sensor against inner and outer disturbance terms. These two controlling loops are being demonstrated as:



Figure 1. Controlling loops block diagram.

The two-DOF gimbal system consists of two subsystems: inner gimbal (elevation axis) and outer gimbal (azimuth axis). The complete scheme of the gimbal system is shown in Figure 2.

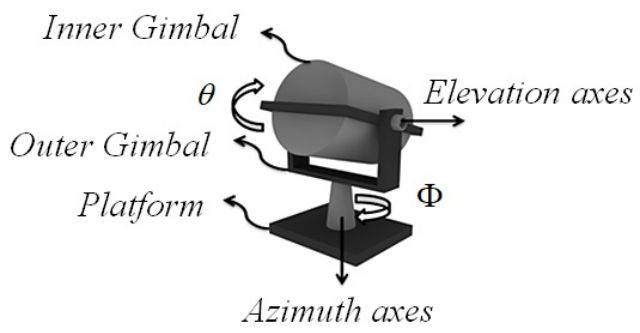

Figure 2. The complete scheme of gimbal system. 
In the above figure, $\varphi$ is the angle around outer gimbal and $\theta$ is the angle around inner gimbal. The deviation in any given direction from a movable target is determined through image processor. The objective is to remove the deviation angle by using $T_{a z}$ and $T_{e l}$ controlling torques.

\section{A. Gimbal system equations}

The mathematical model and the control system related to two axes gimbal system are considered in this section. For this purpose, three reference frames are predicted. Hence, a special angular velocity should be described for each of the systems in every frame.

$$
\omega_{b}=\left[\begin{array}{l}
p \\
q \\
r
\end{array}\right] \omega_{i}=\left[\begin{array}{l}
p_{i} \\
q_{i} \\
r_{i}
\end{array}\right] \omega_{o}=\left[\begin{array}{l}
p_{o} \\
q_{o} \\
r_{o}
\end{array}\right]
$$

where $\omega_{b}, \omega_{i}, \omega_{o}$ indicate the vectors of gimbal body velocity, inner frame velocity, and outer frame velocity respectively. These two systems are established based on enlisting two transformation matrixes indicated below:

$$
\begin{aligned}
L_{o b} & =\left(\begin{array}{ccc}
\cos \varphi & \sin \varphi & 0 \\
-\sin \varphi & \cos \varphi & 0 \\
0 & 0 & 1
\end{array}\right) \\
L_{i o} & =\left(\begin{array}{ccc}
\cos \theta & 0 & -\sin \theta \\
0 & 1 & 0 \\
\sin \theta & 0 & \cos \theta
\end{array}\right)
\end{aligned}
$$

where $L_{o b}$ and $L_{i o}$ are transformation matrixes from body to outer frame and outer frame to inner frame respectively. The inertial matrixes are portrayed in the inner and outer gimbals as:

$$
\begin{aligned}
& J_{i}=\left(\begin{array}{ccc}
J_{i x} & D_{x y} & D_{x z} \\
D_{x y} & J_{i y} & D_{y z} \\
D_{x z} & D_{y z} & J_{i z}
\end{array}\right) \\
& J_{o}=\left(\begin{array}{lll}
J_{o x} & d_{x y} & d_{x z} \\
d_{x y} & J_{o y} & d_{y z} \\
d_{x z} & d_{y z} & J_{o z}
\end{array}\right)
\end{aligned}
$$

where inertial momentum is shown by $J$ and the inertial products for outer and inner gimbals by $d$ and $D$ respectively. From the previously mentioned equations, those ones used for the gimbal system motion in azimuth axis may be calculated by:

$$
\omega_{o}=\left\{\begin{array}{l}
p_{o}=p \cos \varphi+q \sin \varphi \\
q_{o}=-p \sin \varphi+q \cos \varphi \\
r_{o}=r+\dot{\varphi}
\end{array}\right.
$$




$$
\omega_{i}=\left\{\begin{array}{l}
p_{i}=p_{o} \cos \theta-r_{o} \sin \theta \\
q_{i}=q_{o}+\dot{\theta} \\
r_{i}=p_{o} \sin \theta+r_{o} \cos \theta
\end{array}\right.
$$

$B$. The gimbal system equations in the elevation axis

Gimbal system equation in the elevation axis is represented as:

$$
J_{i y} \dot{q}_{i}=T_{e l}+T_{d-e l}
$$

In this equation, $T_{e l}$ and $T_{d-e l}$ function as controlling and undesirable torques respectively and can be computed by equation:

$$
T_{d-e l}=T_{B}+T_{C}
$$

In equation (9), $T_{B}$ and $T_{C}$ represent the values of disturbance torque which could be estimated as:

$$
\begin{aligned}
& T_{B}=-\left(D_{y z} \sin \theta+D_{x y} \cos \theta\right)\left(\dot{p}_{o}+q_{o} r_{o}\right)+\left(D_{y z} \cos \theta-D_{x y} \sin \theta\right) p_{o} q_{o} \\
& +\left[\left(J_{i z}-J_{i x}\right) \cos 2 \theta-2 D_{x z} \sin 2 \theta\right] p_{o} q_{o}-0.5\left[\left(J_{i z}-J_{i x}\right) \sin 2 \theta+2 D_{x z} \cos 2 \theta\right] p_{o}^{2} \\
& T_{C}=\left(D_{x y} \sin \theta-D_{y z} \cos \theta\right) \dot{r}_{o}-0.5\left[\left(J_{i z}-J_{i x}\right) \sin 2 \theta+2 D_{x z} \cos 2 \theta\right] r_{o}^{2}
\end{aligned}
$$

The torque-velocity block diagram of the gimbal system belonging to the elevation axis is presented in Figure 3.



Figure 3. The torque-velocity block diagram in elevation axis.

C. The gimbal system equations in the azimuth axis

Based on Euler equation, the motion equation in azimuth axis can be computed as:

$$
J_{o} \dot{r}_{o}=T_{a z}+T_{d_{1}}+T_{d_{2}}+T_{d_{3}}
$$

In this equation, $J_{o}$ inertial matrix is notably a function of $\theta$ calculated as:

$$
J_{o}=J_{o z}+J_{i x} \sin ^{2} \theta+J_{i z} \cos ^{2} \theta-D_{x z} \sin 2 \theta
$$

In equation (12), $T_{d_{1}}, T_{d_{2}}$ and $T_{d_{3}}$ stand for undesirable disturbances; therefore, $T_{d-a z}$ is the summation of $T_{d_{1}}, T_{d_{2}}$ and $T_{d_{3}}$ representing the disturbance torques.

$$
\begin{aligned}
& T_{d_{1}}=\left[J_{o z}+J_{i x} \cos ^{2} \theta+J_{i z} \sin ^{2} \theta+D_{x z} \cos ^{2} \theta-\left(J_{o y}-J_{i y}\right)\right] p_{o} q_{o} \\
& T_{d_{2}}=-\left[d_{x z}+\left(J_{i z}-J_{i x}\right) \sin \theta \cos \theta+D_{x z} \cos 2 \theta\right]\left(\dot{p}_{o}-q_{o} r_{o}\right)-\left(d_{y z}+D_{y z} \cos \theta\right. \\
& \left.-D_{x y} \sin \theta\right)\left(\dot{q}_{o}+p_{o} r_{o}\right)-\left(d_{x y}+D_{x y} \cos \theta-D_{y z} \sin \theta\right)\left(p_{o}^{2}-q_{o}^{2}\right)
\end{aligned}
$$




$$
\begin{aligned}
& T_{d_{3}}=-\ddot{\theta}\left(D_{x y} \sin \theta-D_{y z} \cos \theta\right)+\dot{\theta}\left[\left(J_{i x}-J_{i z}\right)\left(p_{o} \cos 2 \theta-r_{o} \sin 2 \theta\right)+\right. \\
& \left.+2 D_{x z}\left(p_{o} \sin 2 \theta+r_{o} \cos 2 \theta\right)+\left(D_{y z} \sin \theta+D_{x y} \cos \theta\right)\left(q_{i}+q_{o}\right)-J_{i y} p_{o}\right]
\end{aligned}
$$

The torque-velocity block diagram of the gimbal system related to the azimuth axis is presented in Figure 4.

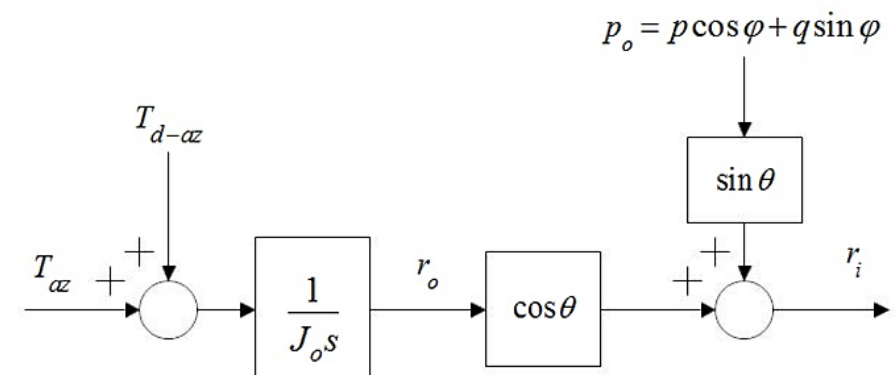

Figure 4. The torque-velocity block diagram for the azimuth axis.

In this block diagram, $r_{i}$ is the output angular velocity in azimuth axis, while $T_{d-a z}$ is the outer disturbance and $T_{a z}$ is the controlling torque.

\section{Design of the direct STR controllers}

The proposed block diagram for the stabilization of two-DOF gimbal system in the azimuth axis is demonstrated in Figure 5. As can be seen, the design appropriately includes both azimuth and elevation axes.



Figure 5. The torque velocity block diagram in elevation axis.

Two different phases are considered for the stabilization of two-DOF gimbal system in this structure. In phase 1, the polynomial parameter estimations in the azimuth and elevation axes are identified through the application of the RLS approach. In phase 2, the control signals are computed and applied to both inner and outer gimbals for the stabilization mode regarding the obtained polynomial.

In the stabilization mode $q_{i}$ and $r_{i}$ stand for angular velocities, while $\theta$ and $\varphi$ are the output angles in the tracking mode. The main function of stabilizer is to vanish the angular velocity regardless of disturbance and keep the non-rotated sensors in the inertial space. Subsequently, gyroscopes are accommodated within the inner gimbal to calculate the angular velocity in both directions. Hence, the suggested RLS approach is applied in discerete-time; the equations of controller design are written to discerete form. To stablish a stable condition, the reference model and closed loop transfer function are considered equal. 


$$
A_{c l}=A_{m}=q^{2}+a_{m_{1}} q+a_{m_{2}}
$$

As for the dynamic equations, the system model is regarded as a second order. Hence, the following reference model is considered for the controller design in the stabilization mode [15]:

$$
\frac{B_{m}}{A_{m}}=\frac{0.4 q^{-1}}{1-0.8 q^{-1}+0.2 q^{-2}}
$$

The parameters of reference model are chosen based on maximom overshot $\left(M_{p}\right)$ and raise time $\left(t_{s}\right)$ as follow:

$$
t_{s}=0.1 \mathrm{sec}, M_{p}=0.05 \%
$$

For controller designing, the polynomial degrees in the stabilization mode are selected as:

$\operatorname{deg}\left(R_{a z}\right)=\operatorname{deg}\left(S_{a z}\right)=1$

In this equation, $R_{a z}(q)$ and $S_{a z}(q)$ polynomials are formulated as:

$$
\begin{aligned}
& R_{a z}(q)=r_{0} q+r_{1} \\
& S_{a z}(q)=s_{0} q+s_{1}
\end{aligned}
$$

where, $r_{0}, r_{1}, s_{0}$ and $s_{1}$ are computed using the RLS algorithm, which is introduced as:

$$
\left\{\begin{array}{l}
P_{a z}^{-1}(k)=P_{a z}^{-1}(k-1)+\phi_{a z}(k) \phi_{a z}^{T}(k) \\
K_{a z}(k)=P_{a z}(k) \phi_{a z}(k) \\
\varepsilon_{a z}(k)=q_{i}(k)-\phi_{a z}^{T}(k) \phi_{a z}(k-1) \\
\omega_{a z}(k)=\omega_{a z}(k-1)+K_{a z}(k)
\end{array}\right.
$$

In this algorithm, $P_{a z}$ is the information matrix, $K_{a z}$ is the weight vector and $\varepsilon_{a z}$ is the error of the parameters. As a consequence, the regressor and parameter vectors for azimuth axis in the stabilization mode are considered as:

$$
\begin{aligned}
& \varphi_{a z}^{T}(k)=\left[T_{a z}(k-1) ; T_{a z}(k-2) ; \theta_{a z}(k-1) ; \theta_{a z}(k-2)\right] \\
& \theta_{a z}=\left[r_{0}, r_{1}, s_{0}, s_{1}\right]
\end{aligned}
$$

After calculating polynomial parameters, $R_{a z}$ and $S_{a z}$ polynomials are specified. Subsequently, $T_{a z}$ control signal for the elevation axis is calculated based on the equation:

$$
T_{a z}=-\frac{S_{a z}}{R_{a z}} r_{i}
$$

\section{Experimental discussion}

Here, we evaluate the suggested controller on the experimental two-DOF gimbal system. The experimental two-DOF gimbal system set-up built consists of a stepper-motor for the azimuth axis and servo-motor for the elevation axis. The servo is operated in torque mode, so the motors act as torque source and they accept an analog voltage as a reference of torque signal. It is equipped with joint position sensors, a Digital Signal Processor (DSP) motion control board, a host computer with CPU core i7 and software environment which generates a user-friendly interface. In our experimental tests, we use the stabilization with a set of two frequencies $\omega_{1}=0.15 \mathrm{rad} / \mathrm{sec}$ and $\omega_{1}=0.5 \mathrm{rad} / \mathrm{sec}$. This experimental two-DOF gimbal system is shown in Figure 6 and this suggested controller is applied to this system which illustrated in Figure 7. The control logic in this research has been implemented on a simple microcontroller board Arduino Uno. Information for the feedback is recivded from the encoder installed on the steppermotor. The calculated errors are used to fit this model to the experimental data. 


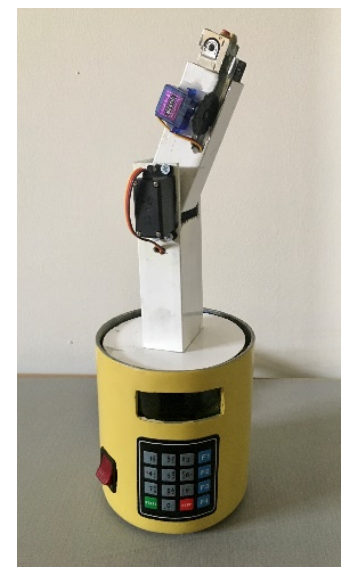

Figure 6. Experimental two-DOF gimbal system

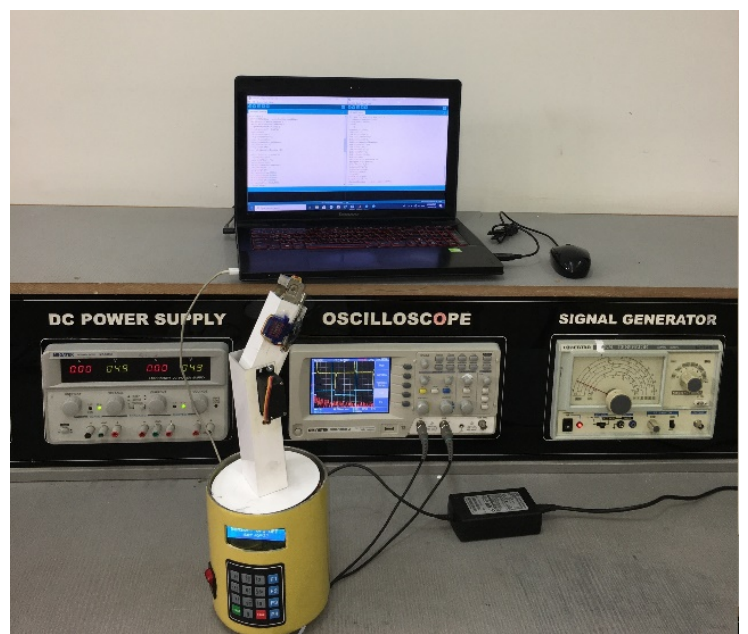

Figure 7. Apply proposed controller to the two-DOF gimbal system in the laboratory

With respect to the output values of the sensor, the model parameters is obtained using RLS algorithm; thus the control response is calculated through direct STR approach and the control torque is obtained and applied to the stepper-motor and servo-motor for both the azimuth and elevation axes, respectively.

\section{Simulation results}

For the purpose of simulating the two-axis gimbal system, the inserted base disturbance to the system is considered as:

$$
\left\{\begin{array}{l}
p=0.5 \sin \left(0.5 \pi t+\frac{\pi}{2}\right) \\
q=0.75 \sin \left(0.25 \pi t+\frac{\pi}{2}\right) \\
r=-0.25 \sin \left(0.5 \pi t+\frac{\pi}{2}\right)
\end{array}\right.
$$

In the aforementioned base angular velocities related to system, the disturbances in both the elevation and azimuth axes are calculated and shown in Figures 8 and 9, respectively. 


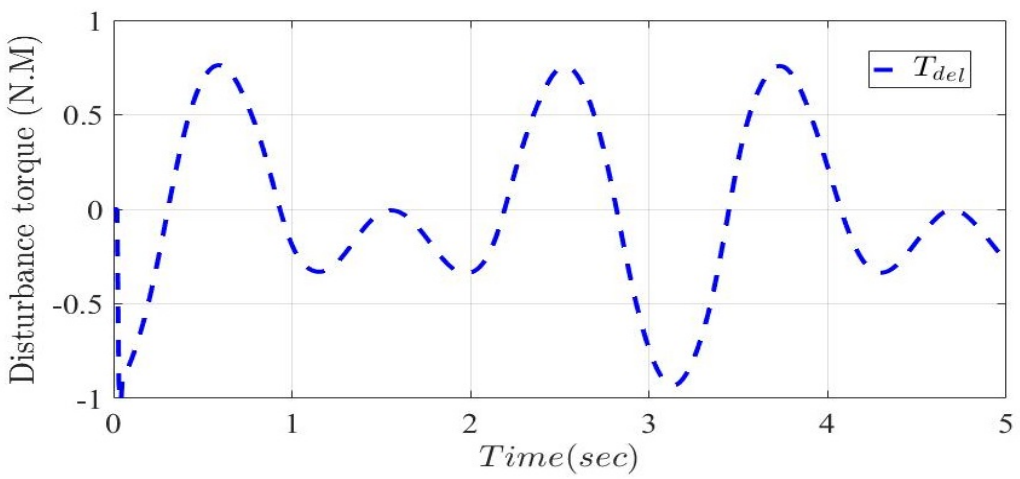

Figure 8 . The disturbance of azimuth gimbal



Figure 9. The disturbance of elevation gimbal.

The inertial momentum for the azimuth axis is represented in Figure 10 demonstrating that the variation has entirely a positive value.

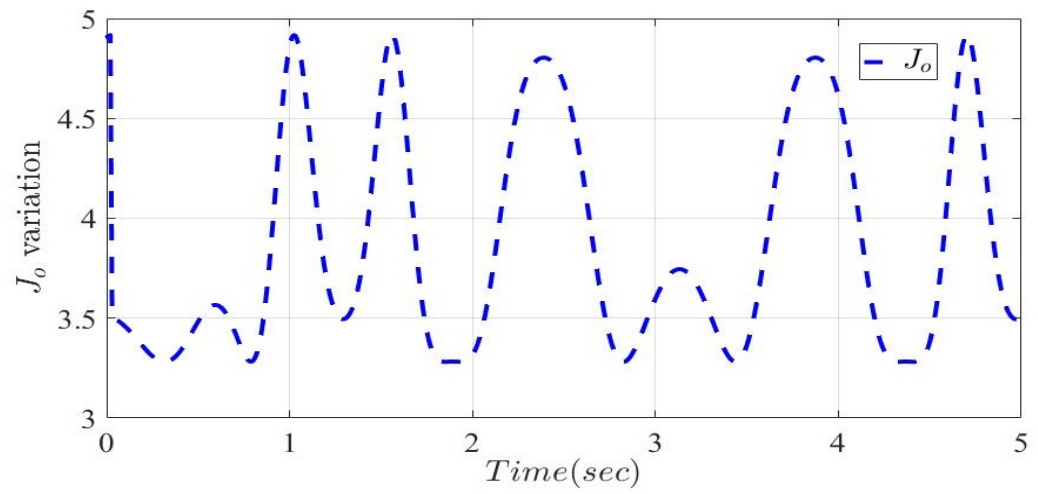

Figure 10. The variation of inertial momentum

The angular velocity for the azimuth axis present in the stabilization mode and its related control signal are represented in Figures 11 and 12, respectively: 


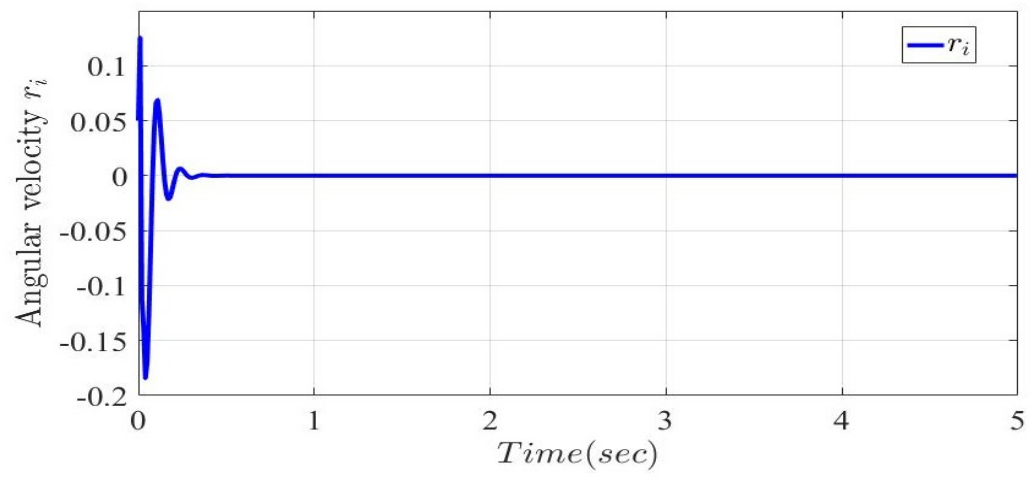

Figure 11. The gimbal stabilization in azimuth axis

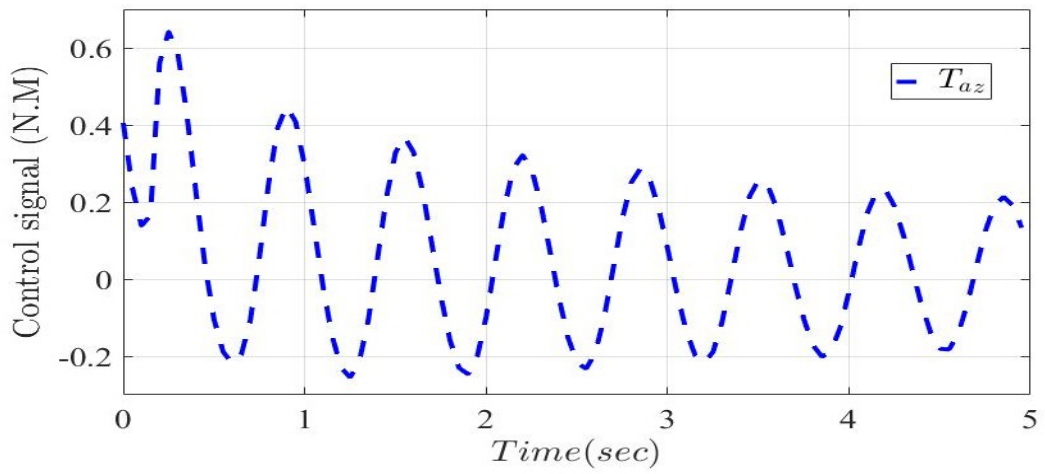

Figure 12. The control signal in stabilization of azimuth axis

The angular velocity of the elevation axis in the stabilization mode and its control signal are shown in Figures 13 and 14 respectively.



Figure 13. The gimbal stabilization in elevation axis 




Figure 14. The control signal in stabilization of elevation axis

Angular velocities in the stabilization loop for azimuth and elevation axes are accompanied with their related control signal for the gimbal system. As observed in Figures 9 and 11, when the system works only in stabilization mode, despite the existing disturbances imposed by the system, the system output remains constant and indirect self-tuning regulator controller satisfactorily eliminates the unwanted impulses.

The parameters estimating the controller polynomials for both elevation and azimuth axes are shown in Figures 15 and 16, respectively. It is obvious that the parameters estimation are convergence to constant value.



Figure 15. The estimation of controller parameters in elevation axis



Figure 16. The estimation of controller parameters in azimuth axis 


\section{Conclusion}

In this article, the two-axis gimbal system was analyzed. For stabilizing a two-axis gimbal system, a direct self-tuning regulator is proposed. Hence, the polynomial parameters in this controller are calculated through RLS approach. Then, the control signal is computed and applied to a two-DOF gimbal system. In the stabilization mode, the proposed controller would remove the disturbances imposed on the system in order to preserve the two-axis gimbal system stability. The designed control signals for both elevation and azimuth axis are smooth and within the range of driver power. To validity the feasibility of the proposed controller, it was tested experimentally by performing an experimental two-axis gimbal system for capturing the response of the control system against different positions.

\section{References}

[1]. Tsai, Nan-Chyuan, Chung-Yang Sue, and Chih-Che Lin. Design and dynamics of an innovative micro gyroscope against coupling effects, Microsystem technologies, vol. 14, no. 3 pp. 295-306, 2008.

[2]. Abdo, Maher, A. Toloei, Ahmad Reza Vali, and Mohammad Reza Arvan. Cascade control system for two axes gimbal system with mass unbalance. International Journal of Scientific \& Engineering Research, vol. 4, no. 9, pp. 903-913, 2013.

[3]. Shuang, Yu, and Zhao Yanzheng. A new measurement method for unbalanced moments in a two-axis gimbaled seeker. Chinese Journal of Aeronautics, vol. 23, no. 1, pp. 117-122, 2010.

[4]. Chen, Xiaocen, and Maoyin Chen. Precise control of a magnetically suspended doublegimbal control moment gyroscope using differential geometry decoupling method. Chinese Journal of Aeronautics, vol. 26, no. 4, pp. 1017-1028, 2013.

[5]. Naderolasli, Amir, and Mohammad Ataei. Applying a direct identifier-based adaptive strategy for enhancing line-of-sight control. Majlesi Journal of Mechatronic Systems, vol. 5, no. 3, 2016.

[6]. Hurák, Zdeněk, and Martin Rezac. Image-based pointing and tracking for inertially stabilized airborne camera platform. IEEE Transactions on Control Systems Technology, vol. 20, no. 5, pp. 1146-1159, 2011.

[7]. Abdo, Maher, Ahmad Reza Vali, Alireza Toloei, and Mohammad Reza Arvan. Research on the cross-coupling of a two axes gimbal system with dynamic unbalance. International Journal of advanced robotic systems, vol. 10, no. 10, pp. 357-370, 2013.

[8]. Kim, S. B., S. H. Kim, and Y. K. Kwak. Robust control for a two-axis gimbaled sensor system with multivariable feedback systems. IET control theory \& applications, vol. 4, no. 4, pp. 539-551, 2010

[9]. Roshdy, Amr A., Chengzhi Su, Hany F. Mokbel, and Tongyu Wang. Design a robust PI controller for line of sight stabilization system, International Journal of Modern Engineering Research, vol. 2, no. 2, pp. 144-148, 2012.

[10]. Naderolasli, Amir, and Mohammad Tabatabaei. Stabilization of the two-axis gimbal system based on an adaptive fractional-order sliding-mode controller, IETE Journal of Research, vol. 63, no. 1, pp. 124-133, 2017.

[11]. Zhou, Xiangyang, Hongyan Zhang, and Ruixia Yu. Decoupling control for two-axis inertially stabilized platform based on an inverse system and internal model control, Mechatronics, vol. 24, no. 8, pp. 1203-1213, 2014.

[12]. Fang, Jiancheng, Rui Yin, and Xusheng Lei. An adaptive decoupling control for three-axis gyro stabilized platform based on neural networks, Mechatronics, vol. 27, pp. 38-46, 2015.

[13]. Qadir, Ashraf, William Semke, and Jeremiah Neubert. Vision based neuro-fuzzy controller for a two axes gimbal system with small UAV, Journal of Intelligent \& Robotic Systems, vol. 74, no. 4, pp. 1029-1047, 2014.

[14]. Naderolasli, Amir, and Mohammad Tabatabaei. Two-axis Gimbal System Stabilization Using Adaptive Feedback Lineari-zation. Recent Advances in Electrical \& Electronic Engineering, vol. 13, no. 3, pp. 355-368, 2020. 
[15]. Naderolasli, Amir, and Mohammad Ataei. Identification of a Two Degree of Freedom Tracker System: Theoretical and Experimental Discussion. Majlesi Journal of Mechatronic Systems, vol. 5, no. 2, pp. 1-6, 2016.

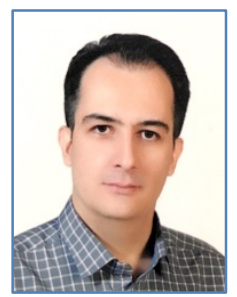

Amir Naderolasli was born in Isfahan, Iran on 1984. He received the B.S. degree and M.S. degree from Islamic Azad University, Isfahan, Iran in 2010 and 2014, respectively. At present, $\mathrm{He}$ is $\mathrm{Ph}$. D. candidate at Najafabad University. His main areas of research interest are stabilization and control of two-DOF gimbal systems, Fractional-order systems and multi agent systems.



Mohammad Ataei (M' 15) received the B.Sc. degree from the Isfahan University of Technology, Iran, in 1994, the M.Sc. degree from the Iran University of Science \& Technology, Iran, in 1997, and Ph.D. from K. N. University of Technology, Iran, in 2004 (joint project with the University of Bremen, Germany) all in control Engineering. He is a professor with the University of Isfahan, Iran. His main areas of research interest are control theory, nonlinear control and chaotic systems' analysis and control. 